\title{
Computational Modeling of Planar Light-Emitting Diode with Mesh-Like Electrode
}

\author{
Yohei Nishidate* \\ The University of Aizu \\ Aizu-Wakamatsu, Japan \\ Julia Kholopova \\ IMT RAS \\ Chernogolovka, 142432, Russia
}

\author{
Valery Zemlyakov \\ National Research University of Electronic Technology \\ Zelenograd, Moscow 124498, Russia
}

\author{
Irina Khmyrova \\ The University of Aizu \\ Aizu-Wakamatsu, Japan \\ Evgeny Polushkin \\ IMT RAS \\ Chernogolovka, 142432, Russia \\ Sergei Shapoval \\ IMT RAS \\ Chernogolovka, 142432, Russia
}

\begin{abstract}
Numerical procedure is developed for modeling of a planar light-emitting diode (LED) with top metal electrode patterned like a mesh with square cross sectional strips. The procedure is applied for modeling LED output performance at different parameters of the mesh-like electrode. Our numerical analysis predicts that the maximum output can be achieved at the mesh opening size around $600 \mathrm{~nm}$.
\end{abstract}

Keywords-light emmitting diode; computational modeling; finite element method; output performance evaluation

\section{INTRODUCTION}

Applications of semiconductor light-emitting diodes (LEDs) in lighting and illumination stimulate intensive research focused on the enhancement of light extraction of the LEDs [1]. Recently, significant enhancement of optical output has been demonstrated for blue GaN/InGaN LED with top metal electrode designed like a mesh [2]. Such patterning of metal electrode results in light emission through the windows in the meshed electrode as shown in Figure 1-(a), where the dark central region corresponds to the solid round shaped nelectrode. The enhancement of optical output was related to potential profile created by meshed electrode in the plane of $\mathrm{p}$ n-heterojunction [3]. It is expected that a peak output can be obtained at a certain range of the mesh pitches

Light generation and extraction in the LED with nonuniform current injection was considered in [4], where the strips of the mesh-like metal electrode were represented by the circular cross sectional wires of finite radius. However, the model has some limitations; in particular, it does not allow to vary individually the width or thickness of the strips. In this paper, we report a numerical procedure and modeling of the LED with the mesh strips having square cross section. The finite element method (FEM) is employed to calculate electric potential distributions. The developed procedure is used to model the spatial distributions of electric potential along the active region and output power density along the mesh openings, and total optical output power at different mesh pitches.
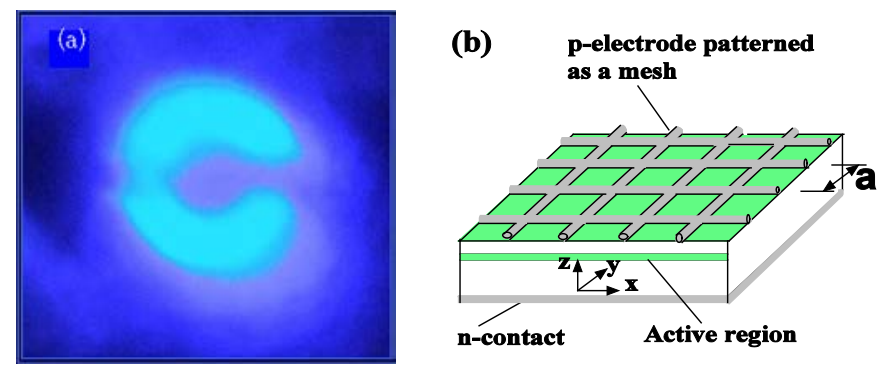

(c)

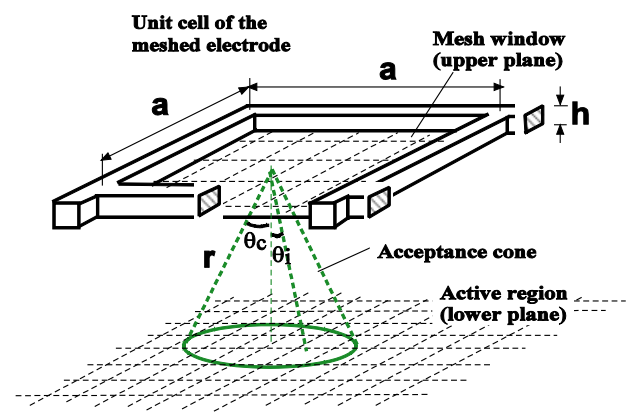

FIGURE I. (A) BLUE LED EMISSION THROUGH THE OPENINGS OF THE MESHED ELECTRODE. (B) SCHEMATIC LED STRUCTURE WITH BOTTOM SOLID N-ELECTRODE AND TOP P-ELECTRODE

PATTERNED AS A MESH. (C) BI-PLANE COMPUTATION MODEL FOR THE UNIT CELL OF THE LED.

\section{OUTPUT POWER OF THE LED}

The power density of light emitted from unit volume with the position $\left(x, y, z_{a c t}\right)$ in the active layer is given by the following equation [5] 


$$
\Delta P\left(x, y, z_{a c t}\right)=\eta \frac{h v}{q \Delta z} J\left(x, y, z_{a c t}\right)
$$

where $z_{a c t}$ is $z$ coordinate of active layer, $\eta$ is the internal quantum efficiency, $h$ is Planck's constant, $v$ is the frequency of output light, $q$ is the electric charge, $\Delta z$ is the thickness of active layer, and $J\left(x, y, z_{a c t}\right)$ is the position-dependent injected current. Therefore, the power density generated in an infinitesimal volume $d V=d x d y d z$ is calculated as follows

$$
\mathrm{d} P\left(x, y, z_{\text {act }}\right)=\eta \frac{h v}{q} J\left(x, y, z_{\text {act }}\right) d x d y
$$

The injected current distribution $J$ is determined by an electric potential distribution $\phi$

$$
J\left(x, y, z_{a c t}\right)=J_{0} \exp \left[\frac{q}{k T} \phi\left(x, y, z_{a c t}\right)\right]
$$

where $k$ and $T$ are Boltzmann constant and the temperature in the unit of Kelvin, respectively. The electric potential distribution can be obtained from a solution of Laplace equation with voltage applied to the meshed top electrodes as boundary conditions. The saturation current density $J_{0}$ may be estimated from a set of physical parameters or from the injected current measured as a current in the external circuit [4].

On the other hand, the power of light which can be extracted from the mesh opening is calculated as the integration of the power from fractional volume in the active layer. The integration should be performed within an acceptance circle since the light is totally internally reflected if the incident angle exceeds a critical angle. Thus, the output power density at the position $\left(x, y, z_{o p n}\right)$ of the mesh opening is calculated as the integration over an acceptance circle

$$
\mathrm{P}\left(x, y, z_{\text {opn }}\right)=\int_{\text {circ }} \frac{\cos \theta_{i}}{4 \pi r^{2}} \mathrm{~T}\left(\theta_{i}\right) d P
$$

where $r$ is the distance between a position on the active layer and a position on the mesh opening of the top metal electrodes, $\theta_{i}$ is the angle of incidence, and $T\left(\theta_{i}\right)$ is the angle dependent transmission coefficient between the semiconductor air interface. The total output power of the system is the multiplication of the number of mesh openings with integration of (4)

$$
\mathrm{P}_{\text {total }}=N_{\text {opn }} \int_{\text {opn }} P\left(x, y, z_{\text {opn }}\right) d x d y
$$

where $N_{\text {opn }}$ is the number of mesh openings (unit cells).

\section{COMPUTATIONAL MODELING}

Our computational modeling procedure consists of the following components:

1. Solve the Laplace equation with boundary conditions by the finite element method to obtain the electric potential.

2. Apply Gauss quadrature rule in a rectangular region (mesh opening) to calculate total output power.

3. Perform Gauss circular rule to supply sampling points for the Gauss quadrature in 2.

4. Construct scattered data interpolation of the finite element solution (electric potential) for sampling of electric potential value at arbitrary position.

\section{A. Finite Element Modeling for Electric Potential Computation}

In previous study, an analytical model for an array of charged parallel wires of circular cross section was used for calculation of potential distributions [2]. More realistic situation of mesh strips with square cross section requires solving three dimensional Laplace equation

$$
\left(\frac{\partial^{2}}{\partial x^{2}}+\frac{\partial^{2}}{\partial y^{2}}+\frac{\partial^{2}}{\partial z^{2}}\right) \phi(x, y, z)=0
$$

with its boundary condition $\left.\phi(\mathrm{x}, \mathrm{y}, \mathrm{z})\right|_{\text {node }}=$ const. applied at the top metal electrodes. The Laplace equation (6) can be rewritten in the weak form via the Galerkin's formulation.

$$
\iiint_{V} \sum_{i=1}^{3} \frac{d[N]^{T}}{d x_{i}} \frac{d[N]}{d x_{i}} d V\{\phi\}=0
$$

Our finite element discretization is realized by the decomposition of field into the three dimensional isoparametric quadratic finite elements.

\section{B. Gauss Circular Rule for Numerical Integration within Acceptance Circles}

The total output power is calculated from equation (6), and hence integration over rectangular mesh opening is required which takes the result of integration within an acceptance circle (4) as its sampling points. The Gauss quadrature rule is a powerful numerical integration method which rapidly approaches to the exact integration. It can be applied for integration over the mesh opening, but integration over the circle is an issue to be considered. It is possible to derive a Gauss circular rule via appropriate transformation of the integration domain.

\section{Moving Least Squares Approximation for Sampling of Electric Potential}

The finite element grid is fixed for calculating electric potential field. It is convenient to construct a scattered data interpolation for resampling the electric field at arbitrary position. For the sake of resampling, the moving least squares (MLS) approximation is employed which is widely used for mesh-less engineering simulations. Our MLS procedure basically follows what briefly explained in literature [6], but the following tri-quadratic basis is used as vector

$$
\boldsymbol{p}^{T}=\left\{1, x, y, z, x y, y z, z x, x^{2}, y^{2}, z^{2}\right\}
$$

For the reconstruction of potential field, 48 closest neighboring points are selected by a bucket search procedure. Since the mesh-like electrode structure is assumed to be periodic, its pattern repeats in lateral directions. Therefore, sampling point coordinates for MLS approximation should be shifted in the multiple of unit cell size when the coordinates are beyond of the cell.

\section{Application for CAlculating Total OUtPut POWER}

\section{A. Problem Description}

The developed procedure is applied to investigate the total output power dependence on unit cell sizes. Figure 2 shows the structure of LED. 


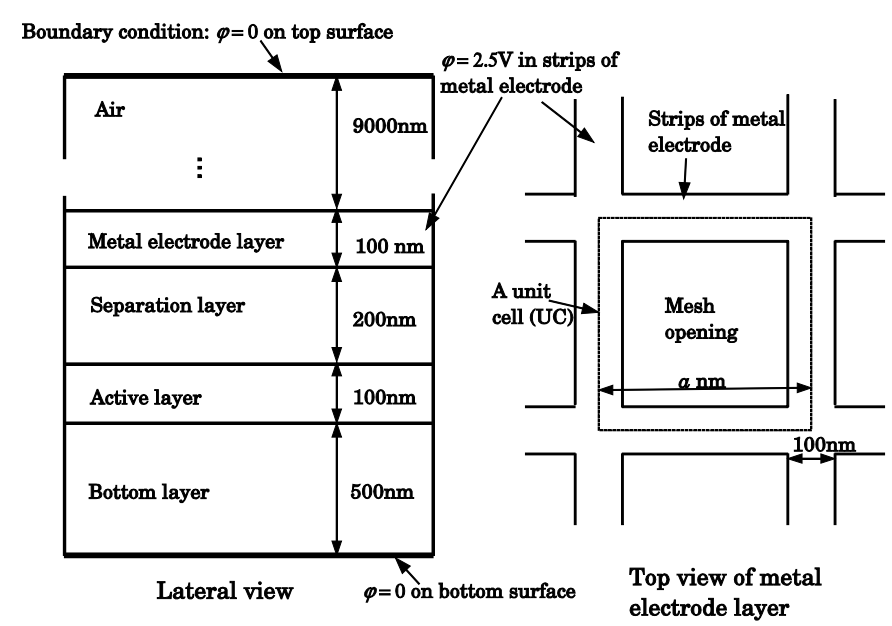

FIGURE II. THE COMPUTATIONAL MODEL OF THE LED. "LATERAL VIEW” SHOWS THE LAYER ARRANGEMENT AND THICKNESS OF EACH LAYER. FROM THE LOWEST LAYER, IT CONSISTS OF

BOTTOM, ACTIVE, SEPARATION, AND METAL ELECTRODE LAYERS, AND THEN VERY THICK AIR LAYER IS PREPARED TO IMITATE NATURAL DECREASING OF POTENTIAL FIELD

The LED is considered as a perfect periodic structure of unit cells, and hence only one unit cell is modeled. The boundary conditions are $\phi=0 \mathrm{~V}$ at the top and the bottom surfaces, and $\phi=2.5 \mathrm{~V}$ in the strips of metal electrode layer. We consider a LED system of total lateral size 36,100 by 36,100 nano meters with varying unit cell size a $=400,500,600$, 800, 900,1000 , and 1200 nano meters (each consists of the number of unit cells $90 \times 90,72 \times 72,60 \times 60,45 \times 45,40 \times 40,36 \times 36$, and $30 \times 30$ ).

All computations are implemented in $\mathrm{C}++$ computer code. The sparse linear systems are solved by preconditioned conjugate gradient method. The largest problem $(\mathrm{a}=1200 \mathrm{~nm})$ consists of 337,280 nodes (degrees of freedom), which takes about 1 minute to solve the equation system, and 25 minutes for calculating total output power, on our ordinary personal computer.

\section{B. Results}

We consider three approaches of total output power calculations: (a) present procedure, (b) present procedure without thick (9000nm) air layer for finite element solution, and (c) present procedure with the analytical potential introduced in [4].

The total output power dependence on the mesh pitch $a$ is shown in Figure 3. It is not realistic to assign boundary condition $\phi=0$ in the distance of zero, and hence the situation (b) with the lowest output power is a reference for comparisons. The predicted total output power is always the largest with our model (a). Also, our model predicts a clear peak of output power depending on the mesh pitch. The peak appears around the mesh pitch $a=600 \mathrm{~nm}$.

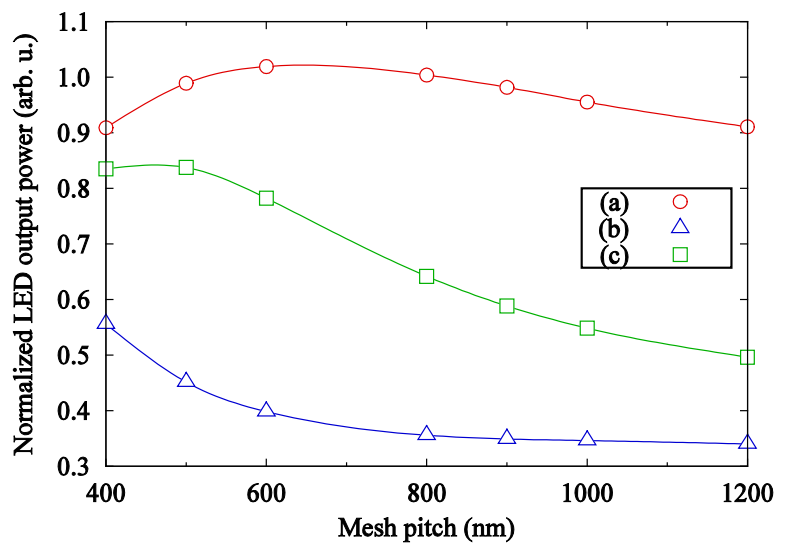

FIGURE III. NORMALIZED TOTAL OUTPUT OPTICAL POWER VERSUS MESH PITCH. CURVES (A) AND (B) CORRESPOND TO SQUARE CROSS SECTIONAL STRIP MODEL. THE DISTANCE BETWEEN TOP SURFACE OF THE LED STRUCTURE AND TOP BOUNDARY OF COMPUTATION DOMAIN IS 9000 NM FOR CURVE (A) AND ZERO FOR CURVE (B). CURVE (C) CORRESPONDS TO CIRCULAR WIRE APPROXIMATION

Figure 4 shows electric potential distributions on the top surface of active layer for unit cell sizes a $=400,800$, and 1200 $\mathrm{nm}$. The distributions obtained by analytical solution tend to be steeper than the finite element solutions, and the surface level (average potential value) is smaller.

Figure 5 shows power density distributions on the mesh opening for unit cell sizes $\mathrm{a}=400,800$, and $1200 \mathrm{~nm}$. The distributions based on the analytical solution is also steeper as the potential distributions, and maximum output power density is larger. However, with our finite element potential calculation, the area in which power density is zero is smaller, which results in the predicting higher total output power. 

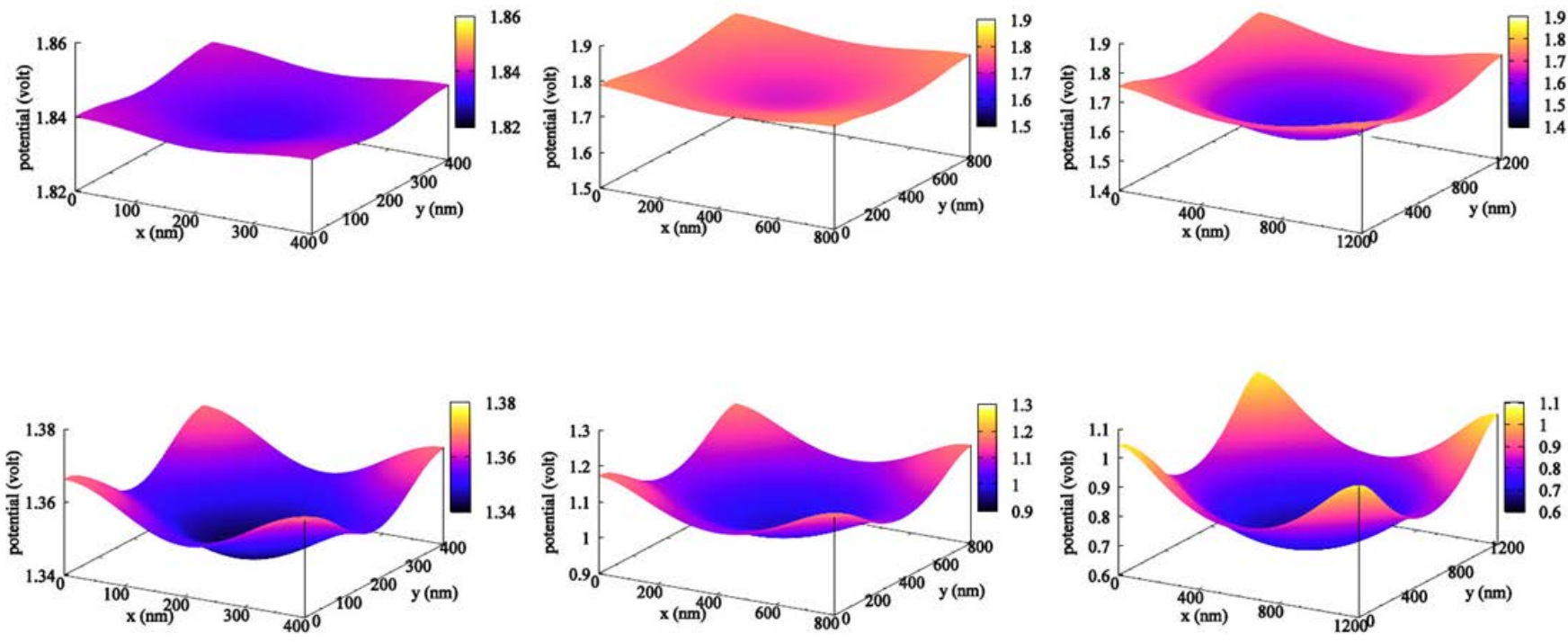

$\mathrm{a}=400 \mathrm{~nm}$

$\mathrm{a}=800 \mathrm{~nm}$

$\mathrm{a}=1200 \mathrm{~nm}$

FIGURE IV. COMPARISONS OF POTENTIAL DISTRIBUTION AT THE ACTIVE LAYER. THE FIRST ROW SHOWS THE DISTRIBUTIONS OBTAINED BY FINITE ELEMENT SOLUTION WITH MLS RESAMPLING, AND THE SECOND ROW SHOWS THOSE OF ANALYTICAL SOLUTION IN [4]
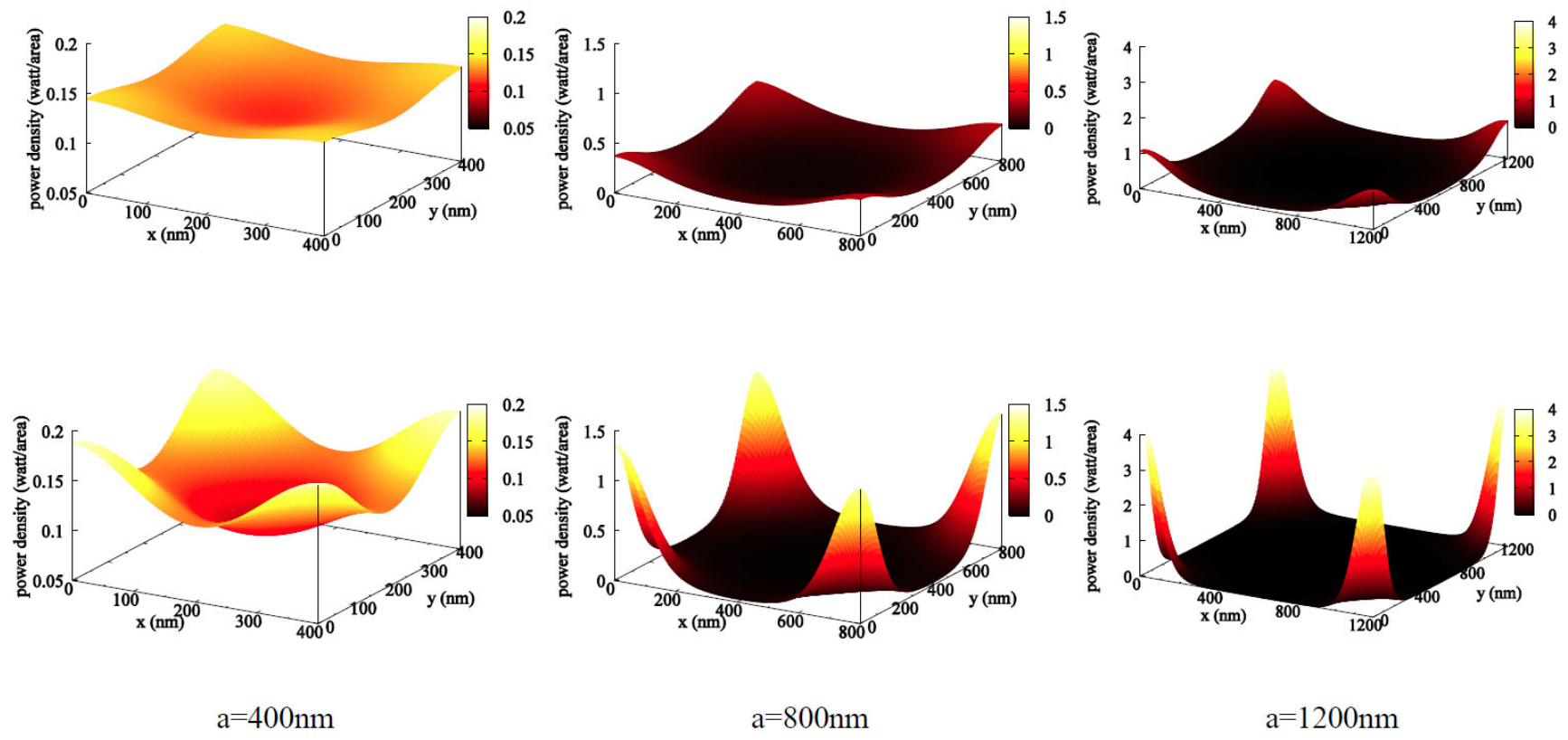

FIGURE V. COMPARISONS OF POWER DENSITY DISTRIBUTION AT THE INTERFACE OF SEPARATION LAYER AND THE TOP METAL ELECTRODE LAYER. THE FIRST ROW SHOWS DISTRIBUTIONS USING PRESENT MODEL, AND THE SECOND ROW SHOWS RESULTS USING [4]

\section{CONCLUSION}

A computational procedure is developed for modeling an LED with mesh like top metal electrodes. The finite element method is applied to obtain the potential energy distribution, and it is resampled by the moving least squares approximation. The resampled potential values are used for integrating over acceptance circles to estimate output power density at mesh opening of top electrode layer of the LED structure.
The procedure is applied for estimating total output power dependence on the unit cell size of mesh opening. The analysis predicts that there is a peak of total output power.

\section{REFERENCES}

[1] S. Nakamura, "The roles of structural imperfections in InGaN-based blue light-emitting diodes and laser diodes", Science vol. 281, 1998, p.956.

[2] S. Shapoval, M. Barabanenkov, V. Sirotkin, E. Polushkin, L. Saptsova, 
A. Kovalchuk, et al., "High efficiency LED with optoelectronically optimized p-contact” Proc. of WOCSDICE 2007, Venice, Italy, 2007, p. 29.

[3] A. Konishi, R. Yamase, I. Khmyrova, J. Kholopova, E. Polushkin, A. Kovalchuk, V. Sirotkin, and S. Shapoval, "Analytical model of lightemitting diodes with patterned contact " Opt. Rev. vol. 20, 2013, p.214.

[4] I. Khmyrova, N. Watanabe, J. Kholopova, A. Kovalchuk, and S. Shapoval, "Light extraction in planar light-emitting diode with nonuniform current injection: model and simulation”, Appl. Opt. vol. 53, 2014, p.4734.

[5] M. Fukuda, Optical Semiconductor Devices, New York: Wiley, 1999.

[6] Y. Nishidate, T. Nagata, S. Morita, and Y. Yamagata, "Ray-tracing method for isotropic inhomogeneous refractive-index media from arbitrary discrete input,” Appl. Opt. vol. 50, 2011, p.5192. 